\title{
3D Face Recognition using Radon Transform and Symbolic LDA
}

\author{
P. S. Hiremath \\ Department of Computer Science \\ Gulbarga University, Gulbarga-585106 \\ Karnataka, India
}

\author{
Manjunatha Hiremath \\ Department of Computer Science \\ Gulbarga University, Gulbarga-585106 \\ Karnataka, India
}

\begin{abstract}
Many recent events, such as terrorist attacks, have exposed the serious weaknesses in most sophisticated security systems. Three dimensional (3D) human face recognition is emerging as a significant biometric technology. Research interest in 3D face recognition has increased during recent years due to the availability of improved 3D acquisition devices and processing algorithms. In this paper, the novel method for three dimensional (3D) face recognition using Radon transform and Symbolic LDA based features of 3D range face images is proposed. In this method, the Symbolic LDA based feature computation takes into account the face image variations to a larger extent and has the advantage of dimensionality reduction. The experimental results have yielded $99.50 \%$ recognition performance with reduced computational cost, which compares well with other state-of-the-art methods.
\end{abstract}

\section{Keywords:}

3D face recognition, range image, radon transform, Symbolic LDA.

\section{INTRODUCTION}

Many recent events, such as terrorist attacks, have exposed the serious weaknesses in most sophisticated security systems. Various government agencies are now more motivated to improve security data systems based on body or behavioral characteristics, often called biometrics. In general, biometric systems process raw data in order to extract a template which is easier to process and store, but carries most of the information needed. It is a very attractive technology, because it can be integrated into any application requiring security or access control, effectively eliminating risks associated with less advanced technologies that are based on what a person has or know rather than whom a person really is. Perhaps the most common biometrics are finger-prints and iris, but many other human characteristics have been studied in last years: finger/palm geometry, voice, signature, face.

With recent advances in image capture techniques and devices, various types of face-image data have been utilized and various algorithms have been developed for each type of an image[1,2]. Among various types of face images, a 2D intensity image has been the most popular and common image data used for face recognition, because it is easy to acquire and utilize. It, however, has the intrinsic problem that it is vulnerable to the change of illumination. Sometimes the change of illumination gives more difference than the change of people, which severely degrades the recognition performance. Therefore, illumination-controlled images are required to avoid such an undesirable situation when $2 \mathrm{D}$ intensity images are used. To overcome the limitation of 2D intensity images, 3D images are being used, such as 3D meshes and range images. A $3 \mathrm{D}$ mesh image is the best $2 \mathrm{D}$ representation of $3 \mathrm{D}$ objects. It contains $3 \mathrm{D}$ structural information of the surface as well as the intensity information of each point. By utilizing the 3D structural information, the problem of vulnerability to the change of illumination can be solved. A 3D mesh image is a suitable image data for face recognition, but the data is complex and difficult to handle. A range image can be a good alternative to a 3D mesh image. A range image contains the structural information of a face and also is simple to utilize for face recognition.

A range image is simply an image with depth information. In other words, a range image is an array of numbers where the numbers quantify the distances from the focal plane of the sensor to the surfaces of objects within the field of view along rays emanating from a regularly spaced grid. For example, a nose tip is the closest point to the camera on a face, so it has the highest numerical value. Range images have some advantages over 2D intensity images and 3D mesh images. First, range images are robust to the change of illumination and color because the value on each point represents the depth value which does not depend on illumination or color. Also, range images are simple representations of 3D information. The 3D information in 3D mesh images is useful in face recognition, but it is difficult to handle. Different from 3D mesh images, it is easy to utilize the 3D information of range images because the 3D information of each point is explicit on a regularly spaced grid. Due to these advantages, range images are very promising in face recognition.

A survey of literature on the research work focusing on various potential problems and challenges in the $3 \mathrm{D}$ face recognition can be found in the survey[1-4,10,11]. Gupta et al.[5] presented a novel anthropometric 3D face recognition algorithm. This approach employs 3D Euclidean and Geodesic distances between 10 automatically located anthropometric facial fiducial points and a linear discriminant classifier with $96.8 \%$ recognition rate. Lu et al.[6] constructed many 3D models as registered templates, and then they matched $2.5 \mathrm{D}$ images (original 3D data) to these models using iterative closest point (ICP). Chang et al. [7] describe a "multi-region" approach to 3D face recognition. It is a type of classifier ensemble approach in which multiple overlapping sub regions around the nose are independently matched using ICP and the results of the 3D matching are fused. The symbolic data analysis[12,13] deals with interval-valued variables, which capture the face image variabilities due to changes in the illumination, orientation and expressions. Hire- 
math and Prabhakar [15] have employed symbolic data analysis approach for $2 \mathrm{D}$ face recognition based on eigenfaces. Hiremath and Manjunath [16] have investigated the 3D face recognition by using Radon Transform and PCA, which yielded recognition accuracy of $95.30 \%$.

In the present paper, the objective is to propose a method for 3D face recognition using Radon transform and Symbolic Linear Discriminant Analysis method (Symbolic LDA). The experimentation is done using the Texas 3D face database [8], and the results demonstrate the efficacy of the proposed method.

\section{MATERIALS AND METHODS}

For experimentation, the Texas 3D Face Database [8] is considered. The 3D models in the Texas 3D Face recognition Database were acquired using an MU-2 stereo imaging system. All subjects were requested to stand at a known distance from the camera system. The stereo system was calibrated against a target image containing a known pattern of dots on a white background. The database contains 1149 3D models of 118 adult human subjects. The number of images of each subject varies from 2 per subject to 89 per subject. The subjects' age ranges from minimum 22 to maximum 77 years. The database includes images of both males and females from the major ethnic groups of Caucasians, Africans, Asians, East-Indians, and Hispanics. The facial expressions present are smiling or talking faces with open/closed mouths and/or closed eyes. The neutral faces are emotionless.

\section{PROPOSED METHODOLOGY}

The proposed method comprises the extension of linear discriminant analysis (LDA) of Radon transformed 3D face range images to the symbolic data framework and is described below.

\subsection{Radon Transform}

The Radon transform (RT) is a fundamental tool in many areas. The 3D Radon transform is defined using 1D projections of a 3D object $f(x, y, z)$ where these projections are obtained by integrating $f(x, y, z)$ on a plane, whose orientation can be described by a unit vector $\vec{\alpha}$. Geometrically, the continuous 3D Radon transform maps a function $R^{3}$ into the set of its plane integrals in $R^{3}$. Given a 3D function $f(\vec{x}) f(x, y, z)$ and a plane whose representation is given using the normal $\vec{\alpha}$ and the distance s of the plane from the origin, the $3 \mathrm{D}$ continuous Radon Transform of $f$ for this plane is defined by

$$
\begin{aligned}
\Re f(\vec{a}, s) & =\int_{-\infty}^{\infty} \int_{-\infty}^{\infty} \int_{-\infty}^{\infty} f(\vec{x}) \delta\left(\vec{x}^{T} \alpha-s\right) d \vec{x} \\
& =\int_{-\infty}^{\infty} \int_{-\infty}^{\infty} \int_{-\infty}^{\infty} f(x, y, z) \delta
\end{aligned}
$$

$(\mathrm{x} \sin \theta \cos \varphi+y \sin \theta \sin \varphi+z \cos \theta-s) d x d y d z$

where $\vec{x}=[x, y, z]^{T}, \vec{\alpha}=[\sin \theta \cos \varphi, \sin \theta \sin \varphi, \cos \theta] \mathrm{T}$, and $\delta$ is Dirac's delta function defined by $\delta(x)=0, x \neq 0$, $\int_{-\infty}^{\infty} \delta(x) d x=1$. The Radon transform maps the spatial domain $(\mathrm{x}, \mathrm{y}, \mathrm{z})$ to the domain $(\vec{\alpha}, s)$, which are not the polar coordinates. The 3D continuous Radon transform satisfies the 3D Fourier slice theorem[9].

\subsection{Symbolic LDA}

In this section, the extension of linear discriminant analysis (LDA) to symbolic data analysis frame work $[12,14,15]$ is considered. Consider the $3 \mathrm{D}$ range face images $\Gamma_{1}, \Gamma_{2}, \ldots, \Gamma_{n}$, each of size $\mathrm{M}$ x N, from Texas 3D range face image database. Let
$\Omega=\Gamma_{1}, \Gamma_{2}, \ldots, \Gamma_{n}$ be the collection of $\mathrm{n} 3 \mathrm{D}$ range face images of the database, which are first order objects. Each object $\Gamma_{l} \in \Omega, l=1,2, \ldots, n$, is described by a matrix $A_{l}$ $(l=1,2, \ldots, n)$, where each component $\tilde{Y}_{a b}, a=1,2, \ldots, M$, and $b=1,2, \ldots, N$, is a single valued variable representing the $3 \mathrm{D}$ range values of the face image $\Gamma_{l}$. An image set is a collection of face images of $m$ different subjects and each subject has different images with varying expressions and illuminations. Thus, there are $\mathrm{m}$ number of second order objects (face classes) denoted by $E=\left\{c_{1}, c_{2}, \ldots, c_{m}\right\}$, each consisting of different individual images, $\Gamma_{l} \in \Omega$, of a subject. The face images of each face class are arranged from right side view to left side view. The feature matrix of $k^{\text {th }}$ sub face class $c_{i}^{k}$ of $i^{t h}$ face class $c_{i}$, where $k=1,2, \ldots, q, i=1,2, \ldots, m$, is described by a matrix $X_{i}^{k}$ of size $M \times N$ that contains interval variable $a_{i a b}^{k}, a=1,2, \ldots, M$, and $b=1,2, \ldots, N$. The matrix is called as symbolic face and is represented as :

$$
X_{i}^{k}=\left[\begin{array}{ccc}
a_{i 11}^{k} & \ldots & a_{i 1 N}^{k} \\
\dot{1} & \cdot & \cdot \\
a_{i M 1}^{k} & \ldots & a_{i M N}^{k}
\end{array}\right]
$$

The interval variable $a_{i a b}^{k}$ of $k^{t h}$ sub face class $c_{i}^{k}$ of $i^{t h}$ face class $c_{i}$ is described as $a_{i a b}^{k}\left(c_{i}^{k}\right)=\left[\underline{x}_{i a b}^{k}, \bar{x}_{i a b}^{k}\right]$, where $\underline{x}_{i a b}^{k}$ and $\bar{x}_{i a b}^{k}$ are minimum and maximum intensity values, respectively, among $(a, b)^{t h}$ feature inside the $k^{t h}$ sub face class of $i^{t h}$ face class. Thus, the $q m$ symbolic faces obtained from the given image database.

Now, apply LDA method to the centers $x_{i a b}^{k} \in R$ of the interval $\left[\underline{x}_{i a b}^{k}, \bar{x}_{i a b}^{k}\right]$ given by

$$
x_{i a b}^{k^{c}}=\frac{\bar{x}_{i a b}^{k}+\underline{x}_{i a b}^{k}}{2}
$$

The $M \times N$ symbolic face $X_{i}^{k^{c}}$ containing the centers $x_{i a b}^{k^{c}} \in R$ of the intervals $a_{i a b}^{k}$ of symbolic face $X_{i}^{k}$ is given by

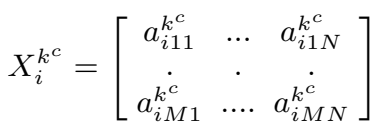

In the symbolic LDA approach, to calculate the scatter (within and between class) matrices of $q m$ symbolic faces $X_{i}^{k}$, where $\mathrm{i}=1,2, \ldots, \mathrm{m}$ and $\mathrm{k}=1,2, \ldots, \mathrm{q}$, the within-class image scatter matrix $S_{w}$ is defined as

$$
S_{w}=\sum_{i=1}^{m} \sum_{k=1}^{q}\left(X_{i}^{k^{c}}-M_{i}\right)^{T}\left(X_{i}^{k^{c}}-M_{i}\right)
$$

where $M_{i}=\frac{1}{q} \sum_{k=1}^{q} X_{i}^{k^{c}}$, and the between-class image scatter matrix $S_{b}$ as

$$
S_{b}=\sum_{i=1}^{m}\left(M_{i}-M\right)^{T}\left(M_{i}-M\right),
$$

where $M=\frac{1}{q m} \sum_{i, k} X_{i}^{k^{c}}$. In discriminant analysis, to determine the projection axis that maximizes the ratio $\frac{\operatorname{det}\left\{S_{b}\right\}}{\operatorname{det}\left\{S_{w}\right\}}$. In other words, to maximize the between-class image scatter matrix while minimizing the within-class image scatter matrix. It has been proved $[10,14]$ that this ratio is maximized when the column vector of projection axis $\mathrm{V}$ is the eigenvector of $S_{w}^{-1} S_{b}$ corresponding to first $p$ largest eigenvalues. For each symbolic face $X_{i}^{k}$, the family of projected feature vectors, $Z_{1}, Z_{2}, \ldots, Z_{p}$ are considered as:

$$
Z_{s}=X_{i}^{k} V_{s}
$$

where $\mathrm{s}=1,2, \ldots, \mathrm{p}$. Let $B_{i}^{k}=\left[Z_{1}, Z_{2}, \ldots, Z_{p}\right]$, which is called as the feature matrix of the symbolic face $X_{i}^{k}$. The feature matrix $B_{\text {test }}$ of the test image $X_{\text {test }}$ is obtained as : 


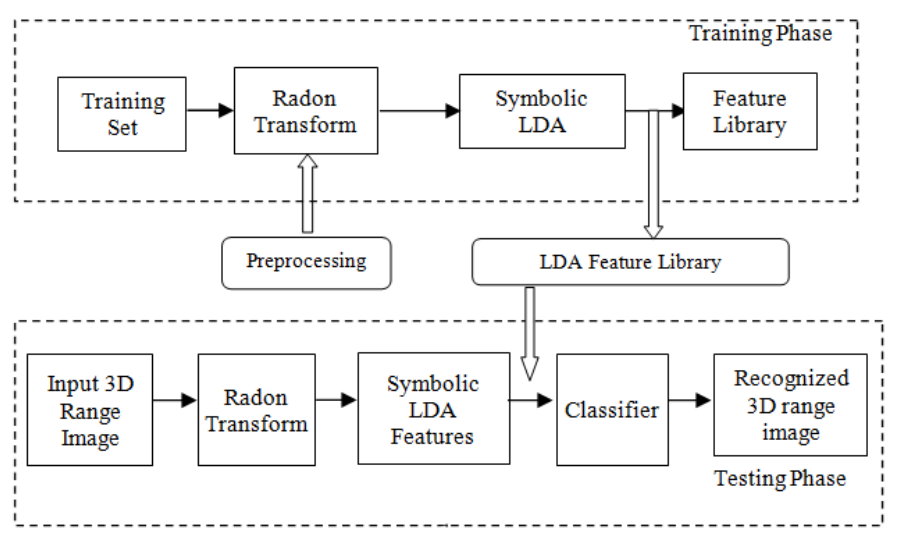

Fig. 1. Overview of proposed framework

$$
Z_{(\text {test }) s}=X_{\text {test }} V_{s},
$$

where $\mathrm{s}=1,2, \ldots, \mathrm{p}$ and

$B_{\text {test }}=\left[Z_{(\text {test }) 1}, Z_{(\text {test }) 2}, \ldots, Z_{(\text {test }) p}\right]$.

\subsection{Proposed Method}

The Figure 1 shows the overview of proposed framework. The algorithms of the training phase and the testing phase of the proposed method are given below:

\section{Algorithm 1: Training Phase}

(1) Input the range image I1 from the training set containing M images.

(2) Apply Radon transform, from $0^{\circ}$ to $180^{\circ}$ orientations (in steps of $h$ ), to the input range image I1 yielding a binary image I2.

(3) Superpose the binary image I2 obtained in the Step 2 on the input range image I1 to obtain the cropped facial range image I3.

(4) Repeat the Steps 1 to 3 for all the $M$ facial range images in the training set.

(5) Apply Symbolic LDA to the set of cropped facial range.

(6) Compute the weights $w_{1}, w_{2}, \ldots, w_{p}$ for each training face image, where $\mathrm{p}<\mathrm{M}$ is the dimension of eigen subspace on which the training face image is projected.

(7) Store the weights $w_{1}, w_{2}, \ldots, w_{p}$ for each training image as its facial features in the Symbolic LDA feature library of the face database.

\section{Algorithm 2: Testing Phase}

(1) Input the test range image $\mathrm{Z1}$.

(2) Apply Radon transform, from $0^{\circ}$ to $180^{\circ}$ orientations (in steps of $h$ ), to the input range image $\mathrm{Z} 1$ yielding a binary image Z2.

(3) Superimpose the binary image $\mathrm{Z} 2$ on $\mathrm{Z} 1$ to obtain the cropped facial image $\mathrm{Z} 3$.

(4) Compute the symbolic weights $w_{i}^{\text {test }}, i=1,2, \ldots, p$, for the test image $\mathrm{Z} 1$ by projecting the test image on the Symbolic LDA feature subspace of dimension $p$.

(5) Compute the Euclidian distance D between the feature vector $w_{i}^{\text {test }}$ and the feature vectors stored in the Symbolic LDA feature library.

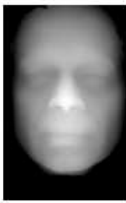

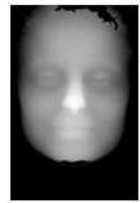

(b)

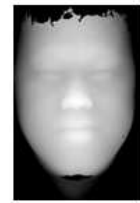

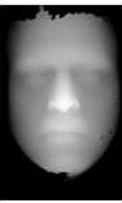

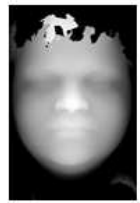

Fig. 2. Sample range images of the training set.

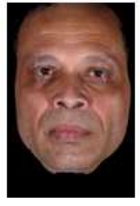

(a)

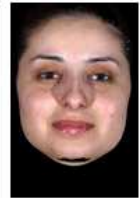

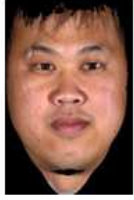

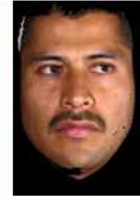

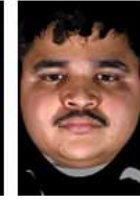

Fig. 3. The facial texture images corresponding to the training range images of the Figure 2

(6) The face image in the face database, corresponding to the minimum distance D computed in the Step 5, is the recognized face

(7) Output the texture face image corresponding to the recognized facial range image of the Step 6.

\section{RESULTS AND DISCUSSION}

For experimentation, the Texas 3D face database [8] is considered. The proposed method is implemented using Intel Core 2 Quad processor @ $2.66 \mathrm{GHz}$ machine and MATLAB 7.9. In the training phase, 10 frontal face images, with different expressions, of each of the 100 subjects are selected as training data set. For each face class (subject), two subclasses are formed; one subclass contains the face images with varying illumination, while the other subclass contains the face images of the same subject with varying facial expressions. In the testing phase, randomly chosen 200 face images of the Texas 3D face database with variations in facial expressions are used. The sample training images which are used for our experimentation are shown in the Figure 2 , and their corresponding texture images are shown in the Figure 3 . The recognition rates obtained by the proposed $(\mathrm{RT}+$ Symbolic LDA) approach is compared with PCA (alone), RT+PCA[16], $\mathrm{RT}+\mathrm{PCA}+\mathrm{LDA}[17]$ and Symbolic PCA[18] methods and the comparison is presented in the Table 1 . The graph of recognition rates versus the number of eigenfaces is shown in the Figure 4 for the proposed method (RT+Symbolic LDA). It is observed that the recognition rate improves as the number of eigenfaces is increased. It is $99.50 \%$ for $5 \mathrm{LDA}$ components in case of the proposed method. Further, the proposed method based on RT and Symbolic LDA outperforms the PCA, RT+PCA, RT+PCA+LDA and RT+Symbolic PCA methods. The RT+Symbolic LDA produces the classification results with same accuracy as that of $\mathrm{RT}+\mathrm{PCA}+\mathrm{LDA}$ is obsereved, but with reduced computational cost

\section{CONCLUSION}

In this paper, a novel method for three dimensional (3D) face recognition using Radon transform and Symbolic LDA based features of 3D range face images is proposed. In this method, the Symbolic LDA based feature computation takes into account face image variations to a larger extent and has advantage of dimensionality reduction. The experimental results have yielded $99.50 \%$ recognition performance with reduced complexity and a 


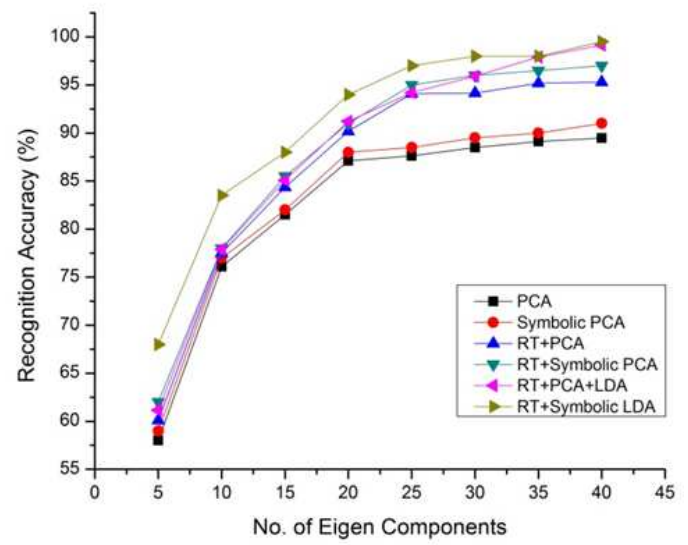

Fig. 4. The recognition accuracy $(\%)$ versus the number of eigenfaces obtained for the proposed method and the other methods.

Table 1. Performance comparison of PCA, Symbolic PCA, RT+PCA and $\mathrm{RT}+$ Symbolic PCA in terms of recognition rates(\%)

\begin{tabular}{|l|l|l|l|l|l|l|}
\hline $\begin{array}{l}\text { No. of } \\
\text { Eigen } \\
\text { Components }\end{array}$ & $\begin{array}{l}\text { PCA } \\
{[16]}\end{array}$ & $\begin{array}{l}\text { Symbolic } \\
\text { PCA } \\
{[18]}\end{array}$ & $\begin{array}{l}\text { RT } \\
\text { PCA } \\
{[16]}\end{array}$ & $\begin{array}{l}\text { RT + } \\
\text { Symbolic } \\
\text { PCA[18] }]\end{array}$ & $\begin{array}{l}\text { RT+PCA } \\
\text { +LDA } \\
{[17]}\end{array}$ & $\begin{array}{l}\text { RT+ } \\
\text { Symbolic } \\
\text { LDA } \\
\text { (Proposed) }\end{array}$ \\
\hline 5 & 58.50 & 59 & 60.10 & 62 & 61.6 & 68 \\
\hline 10 & 76.10 & 77 & 77.50 & 78 & 77.90 & 83.50 \\
\hline 15 & 81.50 & 82 & 84.36 & 85.5 & 85.10 & 88 \\
\hline 20 & 87.10 & 88 & 90.19 & 91 & 91.20 & 94 \\
\hline 25 & 87.61 & 88.5 & 94.10 & 95 & 94.20 & 97 \\
\hline 30 & 88.50 & 89.5 & 94.16 & 96 & 95.91 & 98 \\
\hline 35 & 89.11 & 90 & 95.20 & 96.5 & 97.90 & 98 \\
\hline 40 & 89.47 & 91 & 95.30 & 97 & 99.16 & 99.50 \\
\hline
\end{tabular}

small number of features, which compares well with other stateof-the-art methods. The experimental results demonstrate the efficacy and the robustness of the method to illumination and pose variations. The recognition accuracy can be further improved by considering a larger training set and a better classifier.

\section{REFERENCES}

(1) Kyong I., Chang Kevin, W. Bowyer, Patrick J. Flynn, "Multi-Modal 2D and 3D Biometrics for Face Recognition", Proceedings of the IEEE International Workshop on Analysis and Modeling of Faces and Gestures (AMFG'03), 2003, pp.187-194.

(2) Andrea F. Abate, Michele Nappi, Daniel Riccio, Gabriele Sabatino, "2D and 3D face recognition: A survey", Pattern Recognition Letters, Vol. 28, No. 14, 2007, Pages 18851906.
(3) Shalini Gupta, Mia K. Markey, Alan C. Bovik, "Anthropometric 3D Face Recognition", Int. Journal of Computer Vision, Volu. 90, No. 3, December 2010,pp. 331-349.

(4) Xiaoguang Lu , Dirk Colbry, Anil K. Jain, "Matching 2.5D Scans for Face Recognition", Int. Conf. Pattern Recog. (ICPR 2004), pp.362-366.

(5) Kyong I. Chang, Bowyer, K.W., Flynn P. J., "Adaptive Rigid Multi-region Selection for Handling Expression Variation in 3D Face Recognition", Computer Vision and Pattern Recognition - Workshops, 2005.

(6) S. Gupta, K. R. Castleman, M. K. Markey, A. C. Bovik, "Texas 3D Face Recognition Database", IEEE Southwest Symposium on Image Analysis and Interpretation, May 2010, p 97-100, Austin, TX. URL: http://live.ece.utexas.edu/research/texas3dfr /index.htm.

(7) Amir Averbuch and Yoel Shkolnisky, "3D Fourier based discrete Radon transform", Appl. Comput. Harmon. Anal. 15 , Elsevier Inc., 2003, pp. 33-69.

(8) M. Turk, A. Pentland, "Eigenfaces for Recognition", Journal of Cognitive Neurosicence, Vol. 3, No. 1, 1991, pp. 71-86.

(9) H. Moon, P.J. Phillips, "Computational and Performance aspects of PCA-based Face Recognition Algorithms", Perception, Vol. 30, 2001, pp. 303-321.

(10) Bock, H. H. Diday E. (Eds) : "Analysis of Symbolic Data", Springer Verlag, 2000.

(11) Carlo N. Lauro and Francesco Palumbo, "Principal Component Analysis of Interval Data: a Symbolic Data Analysis Approach", Computational Statistics, Vol.15, No.1, 2000, pp.73-87.

(12) K. Etemad, R. Chellappa, "Discriminant Analysis for Recognition of Human Face Images", Journal of the Optical Society of America, Vol. 14, No. 8, August 1997, pp. 1724-1733.

(13) P. S. Hiremath, C. J. Prabhakar, "Face Recognition Technique Using Symbolic Linear Discriminant Analysis Method", Lecture Notes in Computer Science, Vol. 4338, 2006, pp 641-649.

(14) P. S. Hiremath and Manjunath Hiremath, "3D Face Recognition Using Radon Transform and PCA", International Journal of Graphics \& Image Processing, Vol. 2, No. 2, May 2012. pp. 123-128

(15) P. S. Hiremath and Manjunath Hiremath, "Linear discriminant analysis for 3D face recognition using radon transform", International Conference on Multimedia, Processing, Communication and Computing Applications, 13-15 December 2012 (Accepted).

(16) P. S. Hiremath and Manjunath Hiremath, "3D Face Recognition Using Radon Transform and Symbolic PCA", International Journal of Electronics and Computer Science Engineering, Vol.1, No. 4, October 2012, pp.2342-2349. 